\title{
UTILIZAÇÃO DE ALFENTANIL, SUFENTANIL E FENTANIL EM CÃES ANESTESIADOS COM HALOTANO
}

\author{
USE OF ALFENTANIL, SUFENTANIL AND FENTANYL \\ IN DOGS ANESTHETIZED WITH HALOTHANE
}

\author{
Denise Tabacchi Fantoni ${ }^{1}$ Aline Magalhães Ambrosio ${ }^{2}$ Fábio Futema ${ }^{2}$ \\ Elton Rodrigues Migliati ${ }^{2}$ Eunice Yuriko Tamura ${ }^{2}$
}

\section{RESUMO}

Os analgésicos opióides promovem analgesia intensa, possibilitando o emprego de concentrações reduzidas dos anestésicos inalatórios, minimizando assim a depressão cardiovascular que ocorre durante a anestesia. Vários opióides podem ser empregados na anestesia, sendo que dentre os mais potentes o fentanil, sufentanil e alfentanil são os mais freqüentemente administrados no transoperatório. No presente estudo, comparou-se a ação no sistema cardiovascular desses três agentes durante a anestesia inalatória em cães, submetidos a procedimentos ortopédicos, bem como, avaliou-se a possibilidade de se manter a anestesia com baixas concentrações de halotano. Foram usados três grupos, de dez animais cada, que receberam, após estabilização da anestesia, doses equipotentes de um dos três agentes analgésicos - grupo I - $5 \mu \mathrm{g} / \mathrm{kg}$ de fentanil IV, grupo $I I-1 \mu \mathrm{g} / \mathrm{kg}$ de sufentanil IV e grupo III - $25 \mu \mathrm{g} / \mathrm{kg}$ de alfentanil IV. Os parâmetros cardiovasculares e respiratórios foram avaliados em diferentes tempos de observação. Os resultados obtidos foram analisados através de análise estatística (ANOVA seguida de Dunnett e Bonferroni). Os três agentes promoveram bradicardia importante durante o decorrer do estudo, sendo que apenas o alfentanil promoveu hipotensão significativa após sua administração. Apenas um animal tratado com fentanil apresentou aumento da pressão arterial e freqüência cardíaca durante a anestesia Na recuperação da anestesia, um número maior de animais tratados com alfentanil apresentou sinais de excitação. Através dos resultados obtidos, pode-se concluir que a administração de analgésicos opióides possibilita de fato o emprego de menores concentrações de halotano, promovendo anestesia estável com a manutenção da pressão arterial dentro dos valores normais para a espécie. Dos agentes empregados, o alfentanil é o que promove efeitos cardiovasculares mais pronunciados.

Palavras-chave: fentanil, alfentanil, sufentanil, cão, halotano.

\section{SUMMARY}

Opioid analgesics promote intense analgesia allowing reduction of the concentration of the inhalation anesthetics what minimizes the cardiovascular depression that occurs during anesthesia. Many opioid agents are used during anesthesia. Fentanil, alfentanil and sufentanil are among the most potent agents being preferentially used transoperatively. In the present study, the cardiovascular action of this three agents were compared during inhalation anesthesia in dogs submitted to orthopedic procedures, as well as the possibility of maintenance of anesthesia with halothane in low concentrations. Three groups of ten animals were employed in the experiment. After anesthesia stabilization, animals of group I received intravenously (IV) $5 \mu \mathrm{g} / \mathrm{kg}$ of fentanil, animals of group II $1 \mu \mathrm{g} / \mathrm{kg}$ of sufentanil IV and animals of group III $25 \mu \mathrm{g} / \mathrm{kg}$ of alfentanil IV. Cardiovascular and respiratory parameters were evaluated in various time points. Data were statistically analyzed by means of analyses of variance for repeated measures followed by the Dunnett and Bonferoni post tests.All three agents caused bradicardia during the observation period but only alfentanil promoted significant hypotension. Only one animal that received fentanil showed increase of blood pressure and heart rate simultaneously during anesthesia. At recovery more animals treated with alfentanil showed signs of excitation. With the results obtained the authors could conclude that the use of opioids during anesthesia yield the use of low concentrations of halothane, what contributes with blood pressure maintenance. Among the agents utilized alfentanil promotes more pronounced cardiovascular effects.

Key words: fentanil, alfentanil, sufentanil, dog, halothane.

\section{INTRODUÇÃO}

A anestesia geral no paciente sadio constitui, geralmente, procedimento seguro. Entretanto, a ampla faixa de porte e raças de cães, bem como as diferentes patologias tornam impossível confiar somente em uma técnica de anestesia (HALL \& CLARKE, 1991). Associações de fármacos muito

\footnotetext{
${ }^{1}$ Médico Veterinário, Professor Doutor, Faculdade de Medicina Veterinária e Zootecnia (FMVZ), Universidade de São Paulo (USP), Departamento de Cirurgia. Av. Professor Dr. Orlando Marques de Paiva, 87, Cidade Universitária, Butantã, 05508-900. E-mail: fanauler@uol.com.br. Autor para correspondência.

${ }^{2}$ Médico Veterinário, Aluno de pós-graduação, FMVZ-USP. 
difundidas incluem diferentes grupos farmacológicos, tais como fenotiazínicos, benzodiazepínicos, barbitúricos, agentes dissociativos e analgésicos opióides no preparo e na indução anestésica. Na manutenção da anestesia, tem-se preconizada a utilização de agentes inalatórios halogenados que promovem hipnose e graus variáveis de analgesia e relaxamento muscular sendo, porém, sabidamente depressores cardiovasculares. Deste modo, é de suma importância a redução de suas concentrações durante determinados procedimentos cirúrgicos e/ou nos animais idosos, cardiopatas ou portadores de anomalias, que possam comprometer o desenvolvimento da anestesia. Com a associação de drogas analgésicas, como por exemplo os opióides, esta redução torna-se factível.

O fentanil é opióide primariamente agonista de receptor $\mu$. Como analgésico, estima-se que seja 80 vezes mais potente do que a morfina. Doses elevadas de fentanil produzem intensa rigidez muscular, possivelmente como resultado dos efeitos dos opióides sobre a transmissão dopaminérgica, no corpo estriado, fato que pode comprometer a ventilação no paciente em respiração espontânea (GOODMAN et $\boldsymbol{a l}$., 1987). Analgesia, sedação e depressão respiratória ocorrem após quatro minutos da administração intravenosa com um pico de ação dentro de 10 a 15 minutos, e tempo efetivo de 30 minutos (SOMA \& SHIELDS, 1964). A depressão respiratória ocorre em decorrência ao seu efeito sobre o centro respiratório (GRANDY \& STEFFEY, 1985) observando-se diminuição do volume minuto, o que parece estar relacionado à redução da complacência torácica após administração de altas doses de fentanil (GRANDY \& STEFFEY,1985). Outros efeitos do fentanil são agressividade pós-operatória, apnéia, salivação, bradicardia e relaxamento do esfíncter anal, com ocasional defecação. (SOMA \& SHIELDS, 1964; LUMB \& JONES, 1984).

Análogos do fentanil têm sido desenvolvidos, observando-se variação importante no tempo de ação: de ultracurto (alfentanil), curto (sufentanil) para intermediário/longo (lofentanil) (SHORT, 1987). As vantagens do alfentanil sobre o fentanil e sufentanil são rápido início de ação e menor efeito cumulativo. Já o sufentanil é 5 a 10 vezes mais potente que o fentanil.

McEWAN et al. (1993) e BRUNNER $\boldsymbol{e t}$ al. (1994) relatam diminuição da concentração alveolar mínima (CAM) do isofluorano da ordem de $50 \%$ com fentanil e de 88,6 \% com sufentanil respectivamente. LAKE et al. (1985) obtiveram redução de $48 \%$ da CAM do halotano quando empregaram alfentanil. De fato, estudos em animais mostraram que a CAM dos anestésicos inalatórios é redu- zida de forma mais intensa com sufentanil em relação ao fentanil, morfina ou alfentanil (MURPHY \& HUG, 1982a; MURPHY \& HUG, 1982b; HECKER et al., 1983; MURPHY \& HUG, 1983; LAKE et al., 1985). Em humanos, o mesmo efeito foi observado (SANFORD et al., 1986). Em relação ao alfentanil, a experiência clínica indica que as necessidades de anestésicos voláteis são reduzidas de 30 a $50 \%$ para os primeiros 60 minutos após a indução com este agente.

O halotano promove redução dosedependente do débito cardíaco, da pressão arterial média, do trabalho ventricular, assim como do fluxo sangüíneo coronariano (BEATTIE et al., 1981). O halotano tem fraco poder analgésico (MASSONE, 1994) o que justifica a associação de fármacos analgésicos durante seu emprego. Assim sendo, objetivou-se no presente estudo avaliar comparativamente o emprego de três agentes analgésicos opióides no cão durante a anestesia com halotano, no intuito de se verificar a magnitude das alterações cardiovasculares e a possibilidade de se empregar baixas concentrações do anestésico inalatório.

\section{MATERIAL E MÉTODOS}

Foram utilizados 30 cães machos e fêmeas, sem raça definida, de idade e peso variáveis, ASA I e II submetidos a procedimentos cirúrgicos ortopédicos de duração variada. Os animais foram distribuídos aleatoriamente, mediante sorteio prévio, em três grupos, com dez animais cada. A anestesia foi padronizada em todos os animais e consistiu da administração de acepromazina $(0,1 \mathrm{mg} / \mathrm{kg})$, por via intravenosa (IV), como medicação pré-anestésica. Decorridos 10 minutos, aplicaram-se 5 a $6 \mathrm{mg} / \mathrm{kg}$ de tiopental seguido de midazolam $(0,5 \mathrm{mg} / \mathrm{kg})$, ambos por via IV. Após a indução, o animal foi mantido em anestesia inalatória com halotano. Administrou-se então pancurônio na dose de $0,06 \mathrm{mg} / \mathrm{kg}$ por via IV, sendo instituída ventilação controlada com freqüência respiratória de 12 a 15 movimentos por minuto e volume corrente de $15 \mathrm{ml} / \mathrm{kg}$ em circuito semiaberto. Estes dois parâmetros foram adequados para se manter os valores de dióxido de carbono no ar expirado $\left(\mathrm{EtCO}_{2}\right)$ entre 30 e $45 \mathrm{mmHg}$. O pico de pressão de via aérea foi mantido na faixa de 15 a $20 \mathrm{~cm} \mathrm{H}_{2} 0$. Quatro minutos antes da incisão cutânea, administrou-se um dos três opióides em intervalo de três minutos. Os animais do grupo I receberam fentanil na dose de $5 \mu \mathrm{g} / \mathrm{kg}$, os do grupo II sufentanil na dose de $1 \mu \mathrm{g} / \mathrm{kg}$, e os animais do grupo III alfentanil na dose de $25 \mu \mathrm{g} / \mathrm{kg}$. A partir deste momento, a concentração do anestésico inalatório no ar expirado foi mantida entre 0,4 a $0,6 \%$. A freqüência cardíaca 
(FC) foi obtida através de monitor cardíaco ${ }^{\mathrm{a}}$. As pressões arteriais sistólica, diastólica e média foram avaliadas com a utilização de monitor de pressão arterial não invasivo ${ }^{\mathrm{b}}$. A Saturação da oxihemoglobina foi avaliada através do posicionamento do sensor do oxímetro de pulso ${ }^{c}$ na ponta da língua do animal. A mensuração da concentração do agente anestésico inalado e expirado, assim como do dióxido de carbono do ar expirado (capnografia) foi realizada através do analisador de gases. Todos os parâmetros foram avaliados nos seguintes momentos: após a estabilização da anestesia e início da ventilação mecânica (valor controle) e decorridos 1, 5, 10, 15, 20, 25 e 30 minutos após a aplicação do opióide. A verificação de aumento da pressão arterial e freqüência cardíaca da ordem de $20 \mathrm{mmHg}$ implicaram reaplicação dos agentes.

Os valores obtidos foram avaliados pela análise de variância (ANOVA) para medidas repetidas seguida do Teste de Dunnett para a análise entre os diferentes momentos de cada grupo experimental. Para comparação dos resultados entre os grupos, realizou-se o teste de Bonferroni. O grau de significância estabelecido para os dois testes estatísticos foi de $5 \%(\mathrm{p}<0,05)$.

\section{RESULTADOS}

Verificou-se diminuição estatisticamente significativa da freqüência cardíaca em todos os momentos que se seguiram à administração dos três agentes opióides ( $\mathrm{p}<0,01)$, não havendo restabelecimento da frequiência cardíaca inicial (tabela 1). A FC diminui de forma mais pronunciada com o alfentanil seguida do sufentanil e fentanil, sendo as porcentagens de queda relativas a este fato da ordem de $43,7 \%, 37,6 \%$ e $20,3 \%$, respectivamente no primeiro momento de avaliação. Em relação a análise comparativa entre os grupos, observou-se também uma diminuição mais pronunciada da freqüência cardíaca com alfentanil, quando comparado ao fentanil, evidenciando uma diferença média de 42,20 $\mathrm{bat} / \mathrm{min}$ sendo $\mathrm{p}<0,05$.

Não houve variação estatisticamente significativa nos valores de pressão arterial sistólica, média e diastólica em nenhum momento após a aplicação de fentanil e sufentanil. Fato que não ocorreu com o alfentanil, pois os animais tratados com este agente demonstraram diminuição extremamente significativa dos valores de pressão arterial (sistólica, média e diastólica) da ordem de $30 \%$, um minuto após a sua administração ( $\mathrm{p}<0,01$ ) (tabela 2). No que diz respeito a análise comparativa entre os três grupos estudados, não houve diferença entre os valores da PAS e PAM. No entanto, no que alude à
PAD, foi verificada diferença significativa dos valores entre os animais tratados com fentanil quando comparados àqueles que receberam alfentanil, um minuto após a administração dos agentes, com diferença média de $24,20 \mathrm{mmHg}$, e também entre os animais tratados com sulfentanil e alfentanil um minuto após a administração, sendo a diferença média de 22,80mmHg (figura 1). Um dos animais tratados com fentanil apresentou aumento da freqüência cardíaca e pressão arterial dez minutos após a aplicação. Um animal que recebeu sufentanil e dois que receberam alfentanil necessitaram do emprego de atropina devido à diminuição acentuada da frequiênica cardíaca.

Nenhuma alteração significativa foi observada na análise dos dados referentes aos parâmetros de concentração expirada de dióxido de carbono, saturação de oxigênio, porcentagem inspirada de halotano, porcentagem de halotano no ar expirado, tanto no estudo isolado como na avaliação comparativa entre os grupos.

No que diz respeito à presença de sinais de excitação (meneios de cabeça, movimentos de pedalagem e vocalização) na recuperação anestésica, um animal do grupo fentanil apresentou vocalização e pedalagem. Três animais tratados com alfentanil manifestaram meneios de cabeça e outros dois apresentaram vocalização. Durante o ato cirúrgico, um animal do grupo alfentanil apresentou defecação.

Neste estudo, durante o período de avaliação apenas $20 \%$ dos animais tratados com sufentanil requereram segunda dose deste agente, enquanto que no grupo de animais tratados com fentanil esta taxa foi de $40 \%$ e com alfentanil de $70 \%$. A segunda dose de fentanil e de alfentanil foi administrada em média após 21 minutos da primeira administração, enquanto que com o sufentanil após 26 minutos.

\section{DISCUSSÃO}

Os analgésicos opióides são largamente empregados como coadjuvantes da anestesia geral, com a finalidade de se aumentar a analgesia durante o procedimento cirúrgico, permitindo que se empregue doses reduzidas de anestésicos inalatórios. $\mathrm{O}$ escopo do presente estudo foi a comparação dos efeitos cardiovasculares de três agentes opióides, quais sejam: fentanil, sufentanil e alfentanil, no intuito de se verificar qual deles se adequa mais à prática clínica, uma vez que são escassos os estudos com esses agentes em Medicina Veterinária (THURMON et al. 1996).

O fentanil pode ser empregado em diversas doses na dependência do grau de analgesia/anestesia que se procura. No homem, por exem- 
Tabela 1 - Parâmetros cardiovasculares dos animais tratados com fentanil, sufentanil e alfentanil. São Paulo, 1997.

\begin{tabular}{|c|c|c|c|c|c|c|c|c|c|}
\hline \multirow[b]{2}{*}{ Grupo } & \multirow[b]{2}{*}{ Parâmetro } & \multirow[b]{2}{*}{ controle } & \multicolumn{7}{|c|}{ Minutos após administração opióide } \\
\hline & & & 1 & 5 & 10 & 15 & 20 & 25 & 30 \\
\hline \multirow[t]{2}{*}{ Fentanil } & FC & 143,1 & $114,1 \dagger$ & $114 \dagger$ & $113,3 \dagger$ & $107,3 \dagger$ & $110,6 \dagger$ & $101,9 \dagger$ & $99,5 \dagger$ \\
\hline & & $\pm 29,9$ & $\pm 39,5$ & $\pm 25,2$ & $\pm 28,7$ & $\pm 28,6$ & $\pm 30,2$ & $\pm 27,3$ & $\pm 25,1$ \\
\hline \multirow{2}{*}{ Sufentanil } & FC & 141,5 & $88,5 \dagger$ & $86,7 \dagger$ & $83,9 \dagger$ & $97,8 \dagger$ & $95 \dagger$ & $90,2 \dagger$ & $98,2 \dagger$ \\
\hline & & $\pm 18,7$ & $\pm 23,2$ & $\pm 24,2$ & $\pm 29,2$ & $\pm 38,0$ & $\pm 24,4$ & $\pm 25,4$ & $\pm 27,8$ \\
\hline \multirow[t]{2}{*}{ Alfentanil } & FC & 126,1 & $71,9 \dagger$ & $86,7 \dagger$ & $95,5 \dagger$ & $88,2 \dagger$ & $91,5 \dagger$ & $94,4 \dagger$ & $96,0 \dagger$ \\
\hline & & $\pm 16,0$ & $\pm 22,3$ & $\pm 24,7$ & $\pm 18,6$ & $\pm 22,3$ & $\pm 21,2$ & $\pm 23,9$ & $\pm 36,6$ \\
\hline \multirow[t]{2}{*}{ Fentanil } & PAS & 122,3 & 126,3 & 124,5 & 135 & 127,3 & 128,9 & 127,3 & 128,5 \\
\hline & & $\pm 14,6$ & $\pm 15,5$ & $\pm 13,2$ & $\pm 31,5$ & $\pm 39,3$ & $\pm 30,0$ & $\pm 33,4$ & $\pm 30,4$ \\
\hline \multirow[t]{2}{*}{ Sufentanil } & PAS & 126,3 & 120,5 & 120,9 & 121,1 & 121,5 & 123,6 & 118,4 & 116,2 \\
\hline & & $\pm 29,7$ & $\pm 23,2$ & $\pm 28,3$ & $\pm 30,2$ & $\pm 30,1$ & $\pm 38,3$ & $\pm 42,3$ & $\pm 39,4$ \\
\hline \multirow{2}{*}{ Alfentanil } & PAS & 137 & $110,4 \dagger$ & 120,4 & 126,1 & 128,9 & 134,1 & 130 & 130,1 \\
\hline & & $\pm 19,2$ & $\pm 18,5$ & $\pm 23,3$ & $\pm 19,6$ & $\pm 21,3$ & $\pm 25,2$ & $\pm 21,1$ & $\pm 25,8$ \\
\hline \multirow[t]{2}{*}{ Fentanil } & PAM & 94,8 & 91,7 & 92,8 & 104,7 & 99,5 & 96 & 99 & 94,8 \\
\hline & & $\pm 18,5$ & $\pm 19,8$ & $\pm 16,3$ & $\pm 26,1$ & $\pm 31,0$ & $\pm 23,7$ & $\pm 28,9$ & $\pm 21,3$ \\
\hline \multirow[t]{2}{*}{ Sufentanil } & PAM & 92 & 87 & 89,7 & 90,7 & 92 & 101,7 & 89 & 84 \\
\hline & & $\pm 36,8$ & $\pm 24,1$ & $\pm 23,9$ & $\pm 26,0$ & $\pm 28,2$ & $\pm 31,8$ & \pm 33 & $\pm 26,0$ \\
\hline \multirow[t]{2}{*}{ Alfentanil } & PAM & 105,9 & $70,2 \dagger$ & 86,2 & 92,7 & 91,6 & 97,7 & 90,6 & 86,3 \\
\hline & & $\pm 17,0$ & $\pm 18,0$ & $\pm 2,5$ & $\pm 25,5$ & $\pm 26,2$ & $\pm 24,0$ & $\pm 15,0$ & $\pm 17,7$ \\
\hline \multirow[t]{2}{*}{ Fentanil } & PAD & $78,3^{*}$ & 75,7 & 71 & 84,4 & 83,7 & 79,4 & 79,3 & 76,8 \\
\hline & & $\pm 19,4$ & $\pm 21,4$ & $\pm 15,7$ & $\pm 27,8$ & $\pm 26,6$ & $\pm 18,0$ & $\pm 23,6$ & $\pm 15,2$ \\
\hline \multirow[t]{2}{*}{ Sufentanil } & PAD & $76,1^{*}$ & 71,3 & 71 & 72,4 & 76,5 & 84,9 & 70,1 & 67,6 \\
\hline & & $\pm 33,9$ & $\pm 21,0$ & $\pm 24,2$ & $\pm 23,6$ & $\pm 25,0$ & $\pm 24,7$ & $\pm 27,0$ & $\pm 23,2$ \\
\hline \multirow[t]{2}{*}{ Alfentanil } & PAD & 84,7 & $48,5 \dagger$ & 69 & 77 & 77 & 81,9 & 65,9 & 78,3 \\
\hline & & $\pm 15,2$ & $\pm 17,0$ & $\pm 23,8$ & $\pm 26,9$ & $\pm 32,8$ & $\pm 31,1$ & $\pm 13,7$ & $\pm 31,3$ \\
\hline
\end{tabular}

Valores expressos em médias e desvios-padrões

FC - freqüência cardíaca (batimentos/minuto), PAS pressão arterial sistólica (mmHg), PAM - pressão arterial média (mmHg), PAD - pressão arterial diastólica $(\mathrm{mmHg})$.

* difere significativamente do grupo tratado com alfentanil $(\mathrm{p}<0,05)$.

†difere significativamente do valor controle $(\mathrm{p}<0,05)$.

plo, na anestesia para cirurgia cardíaca, este agente pode ser empregado em doses de até $0,5-0,75 \mathrm{mg} / \mathrm{kg}$ (BOVILL et al., 1984 b) sendo, no caso, usado praticamente como o agente anestésico único. Em contrapartida, no cão, normalmente, é empregado em doses mais baixas: $0,04-0,05 \mathrm{mg} / \mathrm{kg}$ (THURMON $\boldsymbol{e t}$ al., 1996). O sufentanil, sendo 5 a 10 vezes mais potente que o fentanil, é empregado em doses inferiores e, no presente estudo, foi administrado na dose de $0,01 \mathrm{mg} / \mathrm{kg}$, tendo sido a mesma proposta por ERIKSEN et al. (1981). Já o alfentanil possui $1 / 5$ a 1/10 da potência analgésica do fentanil (THURMON et al., 1996) e, portanto, foi empregado na dose de $0,25 \mathrm{mg} / \mathrm{kg}$.

No presente estudo, verificou-se diminuição significativa da freqüência cardíaca nos três grupos experimentais. SOMA \& SHIELDS (1964) observaram bradicardia como consistente ocorrência em cães após injeção IM ou IV de fentanil, tendo sido o mesmo verificado por DOHOO et al. (1985). Essa alteração da frequiência cardíaca é devida ao aumento do tônus vagal (LUMB \& JONES, 1984). A bradicardia verificada no grupo tratado com sufentanil também foi observada por GLENSKI et al. (1988), num estudo em que utilizaram baixas doses de sufentanil como suplemento da anestesia por halotano/ $\mathrm{N}_{2} \mathrm{O}$ em crianças. Os autores relataram significativa diminuição da freqüência cardíaca com $1,5 \mu \mathrm{g} / \mathrm{kg}$ e $1,0 \mu \mathrm{g} / \mathrm{kg}$ após 60 segundos da aplicação do agente, que respondeu prontamente à aplicação de $0,01 \mathrm{mg} / \mathrm{kg}$ de atropina IV. Em cães, ERIKSEN $\boldsymbol{e} t$ al. (1981) verificaram, do mesmo modo, diminuição significativa da freqüência cardíaca (184 bat/min para 90bat/min) após administração de sufentanil. Suspeita-se que o efeito bradicardizante produzido por este fármaco é potencializado também pelo 
Tabela - 2 - Parâmetros de ventilação e oxigenação dos animais tratados com fentanil, sufentanil e alfentanil. São Paulo, 1997.

Minutos após administração do opióide

\begin{tabular}{|c|c|c|c|c|c|c|c|c|c|}
\hline Grupo & Parâmetro & Controle & 1 & 5 & 10 & 15 & 20 & 25 & 30 \\
\hline Fentanil & $\mathrm{EtCO}_{2}$ & $\begin{array}{c}32,3 \\
\pm 10,5\end{array}$ & $\begin{array}{r}36,9 \\
\pm 9,2\end{array}$ & $\begin{array}{l}35,2 \\
\pm 9,7\end{array}$ & $\begin{array}{c}34 \\
\pm 8,8\end{array}$ & $\begin{array}{r}34,6 \\
\pm 9,1\end{array}$ & $\begin{array}{r}33,9 \\
\pm 9,0\end{array}$ & $\begin{array}{r}33,3 \\
\pm 7,6\end{array}$ & $\begin{array}{r}33,2 \\
\pm 8,1\end{array}$ \\
\hline Sufentanil & $\mathrm{EtCO}_{2}$ & $\begin{array}{r}33,5 \\
\pm 6,8\end{array}$ & $\begin{array}{c}33 \\
\pm 7,0\end{array}$ & $\begin{array}{r}32,8 \\
\pm 9,6\end{array}$ & $\begin{array}{r}33,5 \\
\pm 8,2\end{array}$ & $\begin{array}{c}35,9 \\
\pm 8,10\end{array}$ & $\begin{array}{c}35,9 \\
\pm 9,05\end{array}$ & $\begin{array}{c}38,4 \\
\pm 9,03\end{array}$ & $\begin{array}{c}38,2 \\
\pm 2,15\end{array}$ \\
\hline Alfentanil & $\mathrm{EtCO}_{2}$ & $\begin{array}{r}36,9 \\
\pm 5,4\end{array}$ & $\begin{array}{c}34,2 \\
\pm 10,4\end{array}$ & $\begin{array}{c}39,8 \\
\pm 10,3\end{array}$ & $\begin{array}{c}37 \\
\pm 8,7\end{array}$ & $\begin{array}{c}32 \\
\pm 10,3\end{array}$ & $\begin{array}{r}34,8 \\
\pm 9,4\end{array}$ & $\begin{array}{r}33,8 \\
\pm 7,3\end{array}$ & $\begin{array}{r}34,5 \\
\pm 7,3\end{array}$ \\
\hline Fentanil & $\mathrm{Sp} \mathrm{O}_{2}$ & $\begin{array}{r}96,2 \\
\pm 2,8\end{array}$ & $\begin{array}{l}95,5 \\
\pm 2,4\end{array}$ & $\begin{array}{l}96,6 \\
\pm 1,6\end{array}$ & $\begin{array}{c}97,1 \\
\pm 1,6\end{array}$ & $\begin{array}{l}96,8 \\
\pm 1,6\end{array}$ & $\begin{array}{r}96,9 \\
\pm 2,0\end{array}$ & $\begin{array}{l}96,6 \\
\pm 2,2\end{array}$ & $\begin{array}{r}96,7 \\
\pm 1,7\end{array}$ \\
\hline Sufentanil & $\mathrm{Sp} \mathrm{O}_{2}$ & $\begin{array}{r}96,8 \\
\pm 1,5\end{array}$ & $\begin{array}{l}96,7 \\
\pm 1,3\end{array}$ & $\begin{array}{l}96,5 \\
\pm 1,7\end{array}$ & $\begin{array}{r}96,4 \\
\pm 2,7\end{array}$ & $\begin{array}{l}95,6 \\
\pm 2,5\end{array}$ & $\begin{array}{r}95,2 \\
\pm 3,0\end{array}$ & $\begin{array}{l}95,5 \\
\pm 2,5\end{array}$ & $\begin{array}{r}95,8 \\
\pm 2,6\end{array}$ \\
\hline Alfentanil & $\mathrm{Sp} \mathrm{O} \mathrm{O}_{2}$ & $\begin{array}{r}97,4 \\
\pm 1,5\end{array}$ & $\begin{array}{c}96 \\
\pm 2,6\end{array}$ & $\begin{array}{l}96,4 \\
\pm 2,5\end{array}$ & $\begin{array}{l}97,1 \\
\pm 2,0\end{array}$ & $\begin{array}{l}95,9 \\
\pm 2,2\end{array}$ & $\begin{array}{l}95,8 \\
\pm 2,6\end{array}$ & $\begin{array}{l}95,7 \\
\pm 2,6\end{array}$ & $\begin{array}{r}94,8 \\
\pm 2,8\end{array}$ \\
\hline Fentanil & $\%$ Неxp & $\begin{array}{c}0,6 \\
\pm 0,4\end{array}$ & $\begin{array}{c}0,5 \\
\pm 0,2\end{array}$ & $\begin{array}{c}0,4 \\
\pm 0,2\end{array}$ & $\begin{array}{c}0,4 \\
\pm 0,2\end{array}$ & $\begin{array}{c}0,5 \\
\pm 0,2\end{array}$ & $\begin{array}{c}0,5 \\
\pm 0,2\end{array}$ & $\begin{array}{c}0,5 \\
\pm 0,2\end{array}$ & $\begin{array}{c}0,4 \\
\pm 0,2\end{array}$ \\
\hline Sufentanil & $\%$ Неxp & $\begin{array}{r}0,58 \\
\pm 0,6\end{array}$ & $\begin{array}{l}0,48 \\
\pm 0,4\end{array}$ & $\begin{array}{r}0,46 \\
\pm 0,2\end{array}$ & $\begin{array}{r}0,42 \\
\pm 0,2\end{array}$ & $\begin{array}{r}0,39 \\
\pm 0,2\end{array}$ & $\begin{array}{r}0,45 \\
\pm 0,2\end{array}$ & $\begin{array}{l}0,41 \\
\pm 0,1\end{array}$ & $\begin{array}{r}0,42 \\
\pm 0,1\end{array}$ \\
\hline Alfentanil & $\%$ Неxp & $\begin{array}{c}0,5 \\
\pm 0,3\end{array}$ & $\begin{array}{l}0,46 \\
\pm 0,2\end{array}$ & $\begin{array}{l}0,45 \\
\pm 0,2\end{array}$ & $\begin{array}{r}0,42 \\
\pm 0,1\end{array}$ & $\begin{array}{l}0,45 \\
\pm 0,2\end{array}$ & $\begin{array}{l}0,37 \\
\pm 0,1\end{array}$ & $\begin{array}{l}0,52 \\
\pm 0,2\end{array}$ & $\begin{array}{r}0,41 \\
\pm 0,2\end{array}$ \\
\hline
\end{tabular}

Valores expressos em médias e desvios-padrões.

EtCO2 - concentração de dióxido de carbono no ar expirado.

Sp O2 - saturação de oxigênio da hemoglobina.

\% Hexp - concentração de Halotano na expiração.

efeito cronotrópico negativo do halotano (GLENSKI et al., 1988). A administração de sufentanil, em tempo maior (1-2 minutos), parece ocasionar bradicardia de menor intensidade (GLENSKI et al., 1988). No presente estudo, os três agentes opióides foram administrados em intervalo de tempo de três minutos. No entanto, não obstante a observação do período de tempo mencionado, verificou-se queda exacerbada desse parâmetro. Esta redução da frequiência cardíaca após a administração de sufentanil também pode ser resultado da ação colinérgica e ação direta sobre o nó sino-atrial, sendo que o mesmo se aplica ao fentanil (GARDOCKI \& YELNOSKY, 1964; FENNESSY \& RATTRAY, 1971; RIFAT et al., 1971). O efeito bradicardizante do alfentanil verificado neste estudo está em concordância com os achados de KIEN et al. (1986) e BOVILL et al. (1984 b) e ocorre também por ação sobre o vago. No presente estudo, verificou-se que o fármaco que promoveu diminuição da frequiência cardíaca de maior magnitude foi o alfentanil. De fato, a queda com esse agente foi da ordem de $43,7 \%$, enquanto que com o sufentanil a mesma foi de $37,6 \%$ e com o fentanil $20,27 \%$. Uma das possí- veis explicações para este fato seria o menor período de latência do alfentanil (1 minuto) quando comparado ao sufentanil ou fentanil (3 a 5 minutos) (BOVILL et al., 1982; BOVILL et al., 1984 a; THURMON, et al., 1996). A experiência clínica sugere ainda que o sufentanil é 5 a 10 vezes mais potente do que o fentanil, o que, no caso, poderia explicar a diferença verificada entre estes dois últimos agentes no que diz respeito à bradicardia (DE CASTRO, 1976; VAN DE WALLE et al., 1976).

O alfentanil promoveu diminuição extremamente significativa dos valores de pressão arterial média, sistólica e diastólica no presente estudo, um minuto após a sua administração. Esses resultados estão de acordo com aqueles verificados por KIEN et al. (1986), que observaram profundas alterações hemodinâmicas promovidas pelo alfentanil em cães anestesiados por halotano. Esses autores relataram diminuição de $30 \%$ da pressão arterial sistêmica após a dose maciça de alfentanil. No presente estudo, verificou-se queda de $34 \%$, após a administração do mesmo agente. A análise dos dados demonstrou queda significativa da pressão diastólica em proporção maior com alfentanil em relação ao fentanil 


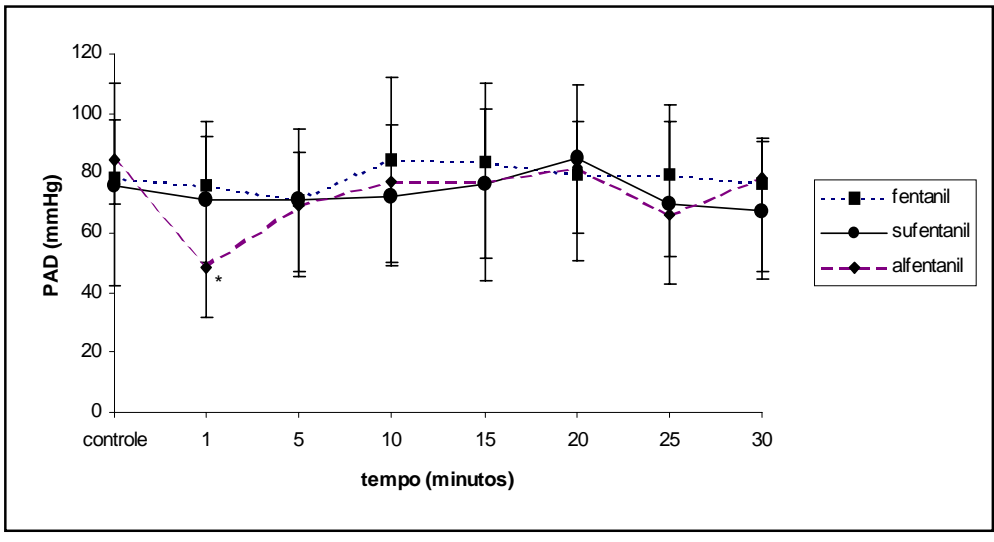

Figura 1 - Variações da pressão arterial diastólica $(\mathrm{mmHg})$ de cães tratados com fentanil, sufentanil ou alfentanil durante o decorrer do tempo. * difere significativamente do grupo tratado com fentanil e sufentanil $(\mathrm{p}<0.05)$
STEFFEY, 1985). A instalação da ventilação mecânica pode desencadear inúmeras alterações hemodinâmicas, refletindo diretamente no débito cardíaco. Estas alterações são determinadas pela pré-carga, pós-carga, contratibilidade cardíaca e frequiência cardíaca (PEREL \& PIZOV, 1992). Essas alterações hemodinâmicas mencionadas não desencadearam alterações nas pressões arteriais, uma vez que os valores controles estavam dentro dos valores normais, e as mensurações posteriores foram nos momentos após a administração de um ou outro opióde já com a instalação da ventilação mecânica.

Verificou-se também que foi (diferença média de $24,20 \mathrm{mmHg}$ ) e ao sufentanil (diferença média de $22,80 \mathrm{mmHg}$ ). Os resultados de freqüência cardíaca, pressão arterial sistólica e diastólica, obtidos no estudo em questão, estão de acordo com aqueles verificados por BOVILL et al. (1984 b). Estes autores avaliaram comparativamente fentanil, sufentanil e alfentanil na anestesia de pacientes que sofreram cirurgia de reposição de válvula cardíaca, onde, com exceção da pressão arterial média e resistência vascular sistêmica, não existiram diferenças significativas nas variáveis hemodinâmicas analisadas nos momentos: pré-indução, indução, intubação, incisão da pele ou após esternotomia. Porém, no que tange a diminuição da pressão arterial média, em ambos os estudos, esta foi mais exacerbada com o uso de alfentanil do que com fentanil. De fato BOVILL et al. (1984 b) verificaram aos 5 minutos de esternotomia, que a pressão arterial média foi significativamente mais elevada no grupo do fentanil. Os mesmos autores relatam também em seu estudo, que 4 pacientes ( 2 do grupo do sufentanil, 1 do grupo fentanil e outro do grupo alfentanil), apresentaram hipotensão (pressão sistólica abaixo de $80 \mathrm{mmHg}$ ) durante a indução da anestesia, e este fato foi associado com a redução de 40 a $60 \%$ na pressão de átrio direito e capilar pulmonar de 25 a $50 \%$ e diminuição no débito cardíaco. No entanto, em ambos os estudos, a hipotensão foi transitória.

Optou-se, neste estudo, pelo uso de ventilação controlada, uma vez que, sabidamente, os analgésicos opióides promovem rigidez muscular com diminuição da complacência torácica e depressão do centro respiratório, que acarretam invariavelmente depressão respiratória com queda do volume minuto e hipercapnia (SOMA \& SHELDS, 1964; LUMB \& JONES, 1984; GRANDY \& possível a manutenção da concentração expirada do halotano em torno de 0,4 a 0,6 ao associar-se os agentes opióides estudados: fentanil, sufentanil e alfentanil, como adjuvantes da anestesia inalatória, uma vez que os parâmetros mensurados mantiveram-se estáveis durante o decorrer do estudo. Apenas um animal que recebeu fentanil apresentou aumento simultâneo de frequiência cardíaca e pressão arterial durante o estudo, o que pode ser indicativo de dor. Outros dois animais de cada grupo apresentaram aumento da pressão arterial isoladamente em apenas um dos momentos de avaliação. Estes fatos sugerem que estes agentes poderiam ser administrados preferencialmente em infusão contínua. O grau de redução da CAM não pode ser estabelecido com acurácia neste tipo de protocolo experimental. Segundo McEWAN et al. (1993), o grau de redução da CAM dos anestésicos voláteis potentes, obtidos após dose bolus de opióide, não pode ser mensurada precisamente, porque a concentração do mesmo é marcadamente dependente da variação entre o tempo de administração do analgésico, sua curva de concentração plasmática e aplicação do estímulo. Observase que uma redução considerável na dose dos anestésicos inalatórios pode ser planejada se eles forem administrados juntamente com o sufentanil ou fentanil e alfentanil (HECKER $\boldsymbol{e}$ t al. 1983; MURPHY \& HUG , 1983; LAKE et al., 1985). Estudos em animais mostram que a CAM dos anestésicos inalatórios é reduzida de forma mais pronunciada com emprego de sufentanil do que fentanil, morfina e alfentanil. Em humanos, o mesmo efeito foi observado por SANFORD et al. (1986). No presente estudo, como os agentes foram empregados em doses equipotentes, não foi possível averiguar este fato.

Quanto ao fato do alfentanil requerer maior número de reaplicações que o fentanil ou sufentanil, configura que este agente é de fato mais 
fraco que os demais enquanto que o sufentanil, o mais potente, requerendo menor número de reaplicações, concordando com os resultados de BOVILL $\boldsymbol{e t}$ al. (1982) e BOVILL et al. (1984b),

A análise dos resultados obtidos permitiu que as seguintes conclusões fossem tecidas: a administração de agentes opióides, durante a anestesia geral com halotano, permite o emprego de baixas concentrações do anestésico inalatório; o alfentanil é o agente opióide que promove efeitos mais pronunciados sobre o sistema cardiovascular, uma vez que se verifica bradicardia e hipotensão após seu emprego. No entanto, o uso dos três agentes é factível durante anestesia geral em cães, uma vez que possibilita manutenção da pressão arterial.

\section{FONTES DE AQUISIÇÃO}

${ }^{a}$ DX 2710 - DIXTAL

${ }^{\mathrm{b}}$ DX 920 - DIXTAL

${ }^{c}$ Modelo 3100 - BCI - Iohchen International - USA

${ }^{\mathrm{d}}$ Multinex - Datascope

\section{REFERÊNCIAS BIBLIOGRÁFICAS}

BEATTIE, C.,TOBB, E.P.,WRIGHT, B. et al. Effects of halothane on coronary blood flow in intact dogs. Anesthesiology, v. 55, n. 3, p. A2, 1981.

BRUNNER, M.D., BRAITHWAITE, P., JHAVERI, R. $\boldsymbol{e} t \boldsymbol{a l}$. MAC reduction of isofluorane by sufentanil. British Journal of Anesthesia, v. 72, p. 42-46, 1994.

BOVILL, J.G., SEBEL, P.S., BLACKBURN, C.L. et al. The pharmacokinetics of alfentanil (R39209): a new opioid analgesic. Anesthesiology, v. 57, p. 439-443, 1982.

BOVILL, J.G., SEBEL, P.S., BLACKBURN, C.L. et al. The pharmacokinetics of sufentanil in surgical patients. Anesthesiology, v. 61, p. 502, 1984a.

BOVILL, J.G., WARREN, P.J., SCHULLER, J.L. et al. Comparison of fentanyl, sufentanil and alfentanil anesthesia in patients undergoing valvular heart surgery. Anesthesia Analgesia, v. 63, p. 1081-1086, 1984 b.

DOHOO, S.E., O'CONNOR, M.K., McDONELL, W.N. et al. A clinical comparison of oxymorfone/acepromazine and fentanyl/ droperidol sedation in dogs. Journal of the American Animal Hospital Association, v. 22, p. 3113-3317, 1985 .

DE CASTRO, J. Pratical applications and limitation of analgesic anesthesia: a review. Acta Anestesilogica Belg, v. 27, p. $107-$ $128,1976$.

ERIKSEN, J.P., BERTHELSEN, N.C., RASMUSSEN, J.P. Early response in central hemodynamics to high doses of sufentanil or morphine in dogs. Acta Anaesthesiologica Scandinavica, v. 25, p. $33-38,1981$.

FENNESSY, M.R., RATTRAY, J.F. Cardiovascular effects of intravenous morphine in the anesthetized rat. European Journal Pharmacology, v. 6, n. 48, p. 1-6, 1971.
GARDOCKI, J.F., YELNOSKY, J.A study of some of the pharmacologic actions od fentanyl citrate. Toxicology Applied Pharmacology, v. 6, p. 48, 1964.

GLENSKI, J., FRIESEN, R.H., LANE, G.A. et al. Low dose sufentanil as a supplement to halothane anesthesia in infants and children. Canadian Journal of Anaesthesia, v. 35, n. 4, p. 379-384, 1988.

GOODMAN, R.M., GILMAN, A.G., RALL, T.W. et al. As bases farmacológicas da terapêutica. 7. ed. Rio de Janeiro: Guanabara, 1987. p. 517.

GRANDY, J.L., STEFFEY, E.P. Anesthesia and the respiratory system. In: SLATTER, D.H. Textbook of small animal surgery. Philadelphia: Saunders, 1985. p. 2621-2633.

HALL, L.W., CLARKE, K.W. General pharmacology of intravenous anaesthetic agents. In. Veterinary anaesthesia. 8. ed. London: Balliére Tindall, 1991. p. 90-97.

HECKER, B.R., LAKE, C.L., DiFAZIO, C.A. et al. The reduction in halothane MAC with sufentanil. Anesthesiology, v. 59, p. $341,1983$.

KIEN, N.D., REITAN, J.A., WHITE, D. et al. Hemodynamic responses to alfentanil in halothane-anesthetized dogs. Anesthesia Analgesia, v. 65, p. 765-770, 1986.

LAKE, C.L., DiFAZIO C.A., MOSCICKI, J.C. Reduction in halothane MAC: comparison of morphine and alfentanil. Anesthesia Analgesia, v. 64, p. 807-814, 1985.

LUMB, W.V. , JONES, E.W. Veterinary anesthesia. 2. ed. Philadelphia: Lea \& Febiger, 1984. 693 p.

McEWAN, A.I., ANAES, F.C., SMITH,C. et al. Isofluorane minimum alveolar concentration reduction by fentanyl. Anesthesiology, v. 78, p. 864-869, 1993.

MASSONE, F. Anestesiologia veterinária - Farmacologia e técnicas. 2. ed. Rio de Janeiro: Guanabara Koogan, 1994. 252 p.

MURPHY, M.R., HUG, C.C. The anesthetic potency of fentanyl in terms of its reduction of enfluorane MAC. Anesthesiology, v. 57, p. $485,1982 \mathrm{a}$.

MURPHY, M.R., HUG, C.C. The enflurane sparing effects of morphine, butorfanol and nalbuphine. Anesthesiology, v. 57, p. $489,1982 b$.

MURPHY, M.R., HUG, C.C. Effects of fentanyl in reducing isoflurane MAC: antagonism by naloxone and nalbuphine. Anesthesiology, v. 58, p. 338, 1983.

PEREL, A., PIZOV, R. Cardiovascular effects of mechanical ventilation. In: PEREL, A., STOCK, M.C. Mechanical vantilatory support. Baltimore: Williams \& Wilkins, 1992. cap. 5 , p. 51-65.

RIFAT, K., MORET, P., GEMPERLE, M. Les effets de l'anesthesie analgesique sequentielle sur le systeme cardiovasculare du chien. Estude experimentale. Ann. Anaesthetic, v. 1, p. 12, 1971.

SANFORD, T.J., SMITH, N.T., DEC-SILVER, H. et al. A comparison of morphine, fentanyl and sufentanil anesthesia for cardiac surgery: induction, emergence and extubation. Anesthesia Analgesia, v. 65, p. 259, 1986. 
SHORT, C.E. Principles \& pratice of veterinary anesthesia Los Angeles: Williams \& Wilkins, 1987. 669 p.

SOMA, L.R., SHIELDS, D. R. Neuroleptoanalgesia produced by fentanyl and droperidol. Journal of American Veterinary Medicine Association, v. 145, n. 9, p. 897-902, 1964

THURMON, J.C., TRANQUILLI, W.J., BENSON, G.J. preanesthetics and anesthetic adjunts. In: LUMB, W.V., JONES, E.W. Veterinary anesthesia. 3. ed. Philadelphia: Lea \& Febiger, 1996. 183-209 p.

VAN DE WALLE, J., LAWERS, P., ADRIAENSEN, H. Duble blind comparison of fentanyl and sufentanil in anesthesia. Acta Anaesthesiologica Belg, v. 27, p. 129-138, 1976

Ciência Rural, v. 29, n. 4, 1999. 